\title{
Natural Language, Knowledge Representation and Discourse
}

James F Allen and Lenhart K Schubert

Department of Computer Science, University of Rochester, Rochester, NY 14627

Goals

Natural language understanding is an area of research that holds great promise for use in intelligent manmachine interfaces and in text analysis and information retrieval. Unfortunately, in most approaches the natural language processing itself is completely separated from the system's general reasoning abilities. This project is investigating several key issues relating to how natural language processing can exploit the system's knowledge representation and reasoning abilities. In particular, we are investigating system architectures specifically aimed at integrating the wide range of language processing and general reasoning modules that are needed to process multisentence language. We are also developing an error-tolerant parser for English that is closely integrated with the system's general reasoning processes. This work is being tested both in the context of narrative understanding, and in the context of man-machine dialog.

More specifically, the principal objectives are (1) to develop a knowledge representation for representing and reasoning about the content of arbitrary English sentences, in the context of a large base of commonsense knowledge; (2) to develop a general, error-tolerant parser for English; (3) to develop a general theory of planning which is suitable for both natural-language systems and robotic systems; and (4) to use this theory of planning to model the content and structure of narratives and dialogs.

\section{Recent Results}

We have developed a new logic of "episodes" that generalizes the notion of events found in many representations based on frames or semantic networks. Within this logic, we are exploring general strategies of input-driven and goal-driven probabilistic inferencing, with the ability to infer explanations and predictions for events and actions (episodes). This work is reported in a paper by Schubert and Hwang, which won an outstanding contribution award at the First International Conference on Knowledge Representation in March 1989.

The parser is based on a variant of GPSG, and uses "preferences" to arrive at preferred interpretations in human-like fashion. Error-tolerance is achieved in part by use of error-tolerant feature unification and by error postulation by the lexical analyzer. A direct, compositional mapping from phrase structure rules to the episodic logic representation is being developed.

We have also developed a new method of identifying the intended speech act in dialog that is significantly more efficient and accurate than previous approaches. This work is reported in the paper in these proceedings.

Plans for the coming year

We plan to further develop the episodic logic, and use it to represent complex situations described in language by tense, aspect and adverbials. A prototype version of the parser will be completed, and the work on speech act modelling will be extended to incorporate more general actions relating to the discourse structure signals in language. 